\title{
Cardiac Expression of Tnnt1 Requires the GATA4-FOG2 Transcription Complex
}

\author{
Nikolay L. Manuylov ${ }^{1}$ and Sergei G. Tevosian ${ }^{1,2, *}$ \\ ${ }^{1}$ Department of Genetics, ${ }^{2}$ Norris Cotton Cancer Center, Dartmouth Medical School, \\ Hanover, $\mathrm{NH}$ \\ E-mail: manuilov@dartmouth.edu; sergei.g.tevosian@dartmouth.edu
}

Received April 29, 2009; Revised June 6, 2009; Accepted June 15, 2009; Published July 4, 2009

\begin{abstract}
Previous work by us and others has shown that the loss of interaction between GATA4 and FOG2 protein partners is embryonic lethal due to heart failure at embryonic day (E) 13.5; however, the role of this important protein duo in various cardiac compartments (e.g., myocardial, endocardial, or epicardial cells) remains to be understood. Although a dual role (both as an activator and a repressor) for the GATA4-FOG2 transcriptional complex has been put forward, the specific genes under GATA4-FOG2 control in the developing heart have remained largely elusive. Since the myocardial-restricted Fog2 reexpression in the Fog2 null embryos is sufficient to extend their life span, identification of GATA4-FOG2 target genes in cardiomyocytes could shed light on the molecular mechanism of GATA4-FOG2 action in these cells. We report here that cardiac expression of slow skeletal troponin $\mathrm{T}$ (Tnnt1) strictly depends on the physical interaction between GATA4-FOG2 in the myocardium of both atria and ventricles.
\end{abstract}

KEYWORDS: Heart, GATA4, FOG2, Zfpm2, Tnnt1

\section{INTRODUCTION}

The multitype, zinc-finger proteins of the FOG (Friend of GATA) family control biological activities of GATA-binding (GATA) transcription factors (for review, see [1,2]). The role for FOG2 (ZFPM2, Mouse Genome Informatics) protein in cardiac development has been firmly established. Initial characterization of the Fog2 gene revealed prominent expression in several developing organ systems (e.g., brain, heart, and gonads)[3,4,5]. Fog $2^{\%}$ (null) embryos die at mid-gestation ( E13.5), with a cardiac defect characterized by a thin ventricular myocardium, common atrioventricular (AV) canal, and the Tetralogy of Fallot malformation[6,7]. Importantly, Fog2 gene loss affects the development of cardiac vasculature[7]. While the formation of an intact epicardial layer and expression of epicardium-specific genes in Fog2 null mutants proceed apparently as normal, markers of cardiac vessel development are not detected[7,8]. Importantly, KDR expression is not detected in the epicardial layer of the Fog2 knockout mice. KDR (FLK1, VEGFR2), the major receptor for VEGF (vascular endothelial growth factor), is an important marker of vascular cells and is absolutely essential for vascular development (e.g., [9], see [10] for a review).

This earlier work drew attention to the role that FOG2 and GATA4 play in the development of the cardiac vascular and epicardial cells. The early demise of the Gata4 null embryos had limited the 
analysis of "Gata4-less" cardiac development to a narrow window between E7.0 and E9.0. Moreover, examination of the Gata4 null mutants did not reveal a substantial down-regulation of any prospective GATA4 target genes and the cardiac manifestation of the knockout (cardia bifida) was attributed to a nonautonomous effect[11,12]. Additionally, as Gata4 $^{-/}$ES cells could contribute to the developing heart and express a wide variety of cardiac markers[13], the significance of GATA4 expression in the cardiac compartment remained uncertain. Understanding the role for the GATA4-FOG2 complex (rather than for each protein separately) was facilitated by generating a Gata4 ${ }^{k i}$ line of mice; "ki" is a V217G mutation in GATA4 that specifically cripples the interaction between GATA4 and FOG proteins[14]. Gata4 ${ }^{k i / k i}$ mutants exhibited a similar (although not identical) phenotype to the Fog 2 null mutants[14]; this work reinforced the importance of the GATA4 protein and the GATA4-FOG2 interaction for both cardiogenesis, and the development of the cardiac vascular and epicardial cells. Reexamination of the cardiac defects in Gata4 chimeric embryos[15], the recent generation of animals with cardiac-limited ablation of Gata4 that have distinct cardiac-specific defects[16,17], as well as a report connecting mutations in the GATA4 gene to congenital heart defects in humans[18], further confirmed the pivotal role for GATA4 in cardiogenesis.

The absence of the GATA4-FOG2 complex is embryonic lethal at E13.0-13.5 due to heart failure; however, the mechanism by which GATA4-FOG2 interaction loss translates into heart failure remains to be understood. Given that myocardial-restricted Fog2 re-expression is sufficient to rescue cardiac vascular development and extend the life span of the Fog2-null embryos[7,19], we reasoned that GATA4FOG2 target genes should exist in the myocardium. We have now identified the skeletal troponin Tnnt1 gene as a myocardial target of the GATA4-FOG2 transcriptional complex. This finding was unexpected, since Tnnt1 is mostly expressed in skeletal muscle where TNNT1 forms a part of the skeletal troponintropomyosin complex. Now our data demonstrate that cardiac expression of Tnnt1 requires GATA4FOG2 interaction.

\section{EXPERIMENTAL PROCEDURES}

\section{Animals}

Fog 2 heterozygous and Gata $^{k i}$ heterozygous animals were crossed to generate Fog $2^{-/}$and Gata4 ${ }^{k i / k i}$ (both mixed 129xC56BL/6 background); the generation and genotyping of Fog2 transgenic, Fog2- and Gata4targeted animals have been previously described[7,14]. Mhc-Fog2 transgenic mice[7] were genotyped with primers specific for Fog2 cDNA P3 5'-CAACTGCATTGTGTACAGC-3' and P8 5'GCTCTTGGTGCATTGTGGGAAG-3'.

\section{Affymetrix Microarray Analysis of Gene Expression}

Embryonic hearts were dissected from E12.5 wild-type and Gata4 ${ }^{k i k i}$ or Fog2 ${ }^{-/}$mutant embryos and transferred to RNAlater solution (Ambion). RNA was isolated with an RNeasy Mini Kit (Qiagen) by standard protocol and subsequently treated with DNAseI (Roche) to remove any possible DNA contamination. DNAseI was heat-inactivated for $15 \mathrm{~min}$ at $70^{\circ} \mathrm{C}$, and RNA was precipitated by standard protocol and diluted in $20 \mu \mathrm{H} \mathrm{H}_{2} \mathrm{O}$. Affymetrix oligonucleotide arrays were used for RNA expression analysis[20,21]. The array experiment was performed by the Dartmouth Genomic and Microarray Laboratory, according to the standard protocol. The microarray data have been deposited at the GEO database GSE14906 and were analyzed using Gene Traffic Software (Iobion Informatics). 


\section{Transgenic Mice}

A 5' 2.4-kb Tnnt1 mouse genomic fragment was obtained by PCR using primers tnnt1_2.4F 5'AAGTTTGAGGGCTGAGCCAT-3' and tnnt1pR GGCTGGGTCCACAGATGCTGTA; the conserved fifth intron of the mouse Tnntl gene was similarly generated with primers tnntliF 5'TTGAACTCATAGCAACTCTC-3' and tnnt1_2.4R 5'-TTAAGAGTTAAGGTTGGCTG-3'. To identify cis-regulatory elements responsible for cardiac and skeletal muscle expression of Tnnt1, we fused the 5' fragment upstream of an ATG codone of the lacZ reporter gene in pSDKlacZpA (a kind gift of Janet Rossant), while the intron sequence was subsequently inserted 3' to the LacZ-SV40 polyA sequences to generate pTnnt1-LacZpa. The fragment of pTnnt1-LacZpa containing the Tnnt1 regulatory sequences directing expression from the LacZ-pA reporter was isolated using standard methods[22]. Transgenic animals were generated by the Dartmouth Transgenic and Genetic Construct Shared Resource Center by pronuclear injection into fertilized eggs. $\mathrm{F}_{0}$ embryos were collected and analyzed at E12.5 by an X-gal staining assay.

\section{Whole Mount In Situ Hybridization}

Embryos at various stages were removed from the uterus and their internal organs were removed to expose the heart; alternatively, whole embryonic hearts were dissected out. Embryonic tissues were fixed with $4 \%$ paraformaldehyde (PFA) in $1 \times \mathrm{PBS}$ at $4^{\circ} \mathrm{C}$ overnight. Further processing of embryos and in situ hybridization analysis were carried out essentially as described[23]. Tnnt1 and Tnnt2 dig-labeled RNA probes have been generated by RT-PCR from E12.5 hearts. To generate the Tnnt1 probe, we used primers tnnt $1 p F$ 5'-GGTCAAGGCAGAACAGAAGC-3' and tnnt1pR 5'-CTCCACACAGCAGGTCATGT-3'; Tnnt2 probe primers are tnnt $2 p F$ 5'-CGGAAGAGTGGGAAGAGACA and tnnt2pR 5'AGCTAAGCCAGCTCCCACTA-3'. Hearts were photographed and images were processed and assembled as previously described[22,24].

\section{Quantitative RT-PCR Analysis}

The hearts (ventricles and atriums) were dissected in PBS from E12.5 or E17.5 embryos, and transferred in RNAlater solution (Ambion). RNA was isolated with an RNeasy Mini Kit (Qiagen) in $30 \mu$ l of RNAsefree TE buffer. During isolation, RNA samples were treated with RNAse-Free DNAse set for 15 min on RNeasy columns (Qiagen), according to the manufacturer's instructions. Each sample was divided into two aliquots, one of which was reverse transcribed using the M-MLV reverse transcriptase (Invitrogen), following the manufacturer's instructions. The second aliquot was used as a control without reverse transcription to identify and discard samples with DNA contamination. All real-time PCR assays were carried out using SYBR Green Universal PCR Master Mix (Applied Biosystems). The PCR reactions contained $25 \mathrm{ng}$ of cDNA and gene-specific primers at a final concentration of $1 \mu M$ each. The assays were run under standard SYBR Green conditions on the ABI 7500 instrument. A standard curve for each gene was generated using serial dilutions of cDNA. Relative expression levels for each sample were determined in the same run and were expressed as the ratio of the RNA amount (of interest) to the amount of control RNA (Gapdh). SYBR Green reactions were performed in duplicates and the experiments were repeated independently at least three times (for at least three samples). Gene-specific primers were designed using the Primer Express software (Perkin Elmer Life Sciences), namely:

Gapdh-qRT_F 5'-GCTCACTGGCATGGCCTTCCGTG-3';

Gapdh-qRT_R 5'-TGGAAGAGTGGGAGTTGCTGTTGA-3';

Tnnt1-qRT_F 5'-GGTCAAGGCAGAACAGAAGC-3'; and

Tnnt1-qRT_R 5'- GCGGTTGTAGAGCACATTGA-3' 


\section{B-Galactosidase Assay}

Embryos were fixed and stained using X-gal essentially as previously described[22]. The staining was continuously monitored until a satisfactory color development was achieved (2-5h). Embryos were then fixed overnight in 4\% PFA in PBS and photographed as previously described[22].

\section{RESULTS}

\section{Microarray Analysis of RNA Expression in FOG2 and GATA4 Mutant Hearts}

In order to identify the targets of GATA4-FOG2 action in mammalian heart development, we performed Affymetrix microarray comparisons of gene expression in normal and mutant hearts at E12.5. We compared RNA samples from both Fog2 null and Gata4 ${ }^{k / / k i}$ mutant E12.5 hearts to the wild-type control E12.5 hearts. We reasoned that as the phenotypes of the Fog2 knockout and Gata4 ${ }^{k i k i}$ mutation are similar[7,14], we should expect to identify a similar set of differentially expressed genes in both experiments. As an additional control, we expected to find the Fog2 gene expression absent in the mutant (null) Fog 2 cardiac sample, but not Gata4 $^{k i k i}$ sample.

The microarray profiling yielded surprisingly few gene sets that were differentially represented $(\sim 2.5$ times up- or down-regulated) in the mutant samples vs. controls. Importantly, the results were consistent between "Fog2" and "Gata4 ${ }^{k i}$ " experiments (similar gene sets were recovered), with the exception of the gene set corresponding to the Fog 2 gene that was absent in the Fog 2 mutant sample, as we had predicted (Supplemental Table 1; see also [25]). The results of the microarray experiment are available at the GEO database (GSE14906).

\section{Tnnt1 is a Target of the GATA4-FOG2 Transcription Complex}

Microarray experiments have identified Tnnt1 as a target of GATA4-FOG2 activation in the heart. Based on the microarray data, the expression of Tnnt1 was down-regulated $\sim 5$ times in the Fog2-null sample and $\sim 7$ times in the Gata ${ }^{k i k i}$ sample; Tnntl was the "most down-regulated" gene in both mutants (see also Supplementary Table in Smagulova et al.[25]). Tnnt1 was also the only gene of the troponin group that was down-regulated in the Gata4 and Fog2 mutant hearts; other troponins (e.g., Tnnil, Tnnc1, or cardiacrestricted Tnnt2 and Tnni3) were expressed at a similar level in control and mutant GATA4/FOG2 samples (not shown). Given this strong dependence of cardiac Tnnt1 expression on the GATA4-FOG2 interaction, we decided to pursue the analysis of Tnnt 1 expression further.

\section{Tnnt1 Expression in Cardiac Development}

Expression of the Tnnt1 gene in the rodent heart has been previously documented[26,27] and is consistent with our data. The whole-mount in situ hybridization (WISH) experiment using anti-Tnnt1 RNA as a probe demonstrated that, at E9.5, the embryonic heart of a mouse is positive for Tnnt1, with expression visible in the outflow track and in the forming interventricular groove (Fig. 1A,B). At E10.5, Tnntl expression expands posteriorly towards the apex; the expression also appears in the left ventricle (Fig. 1C-E). However, at E11.5, the expression in the outflow track is down-regulated and by E12, the expression is mostly confined to the ventral interventricular groove with some expression in the left ventricle; from about E12.0, the outflow track cells are negative for Tnnt1 (Fig. 1F). In the E12.5 heart, interventricular Tnnt1 expression expands laterally and by E14.5, the gene is expressed throughout the ventral side of the left ventricle; in the right ventricle, the expression is enhanced in the apical region, while the cells in the outflow 
E9.5
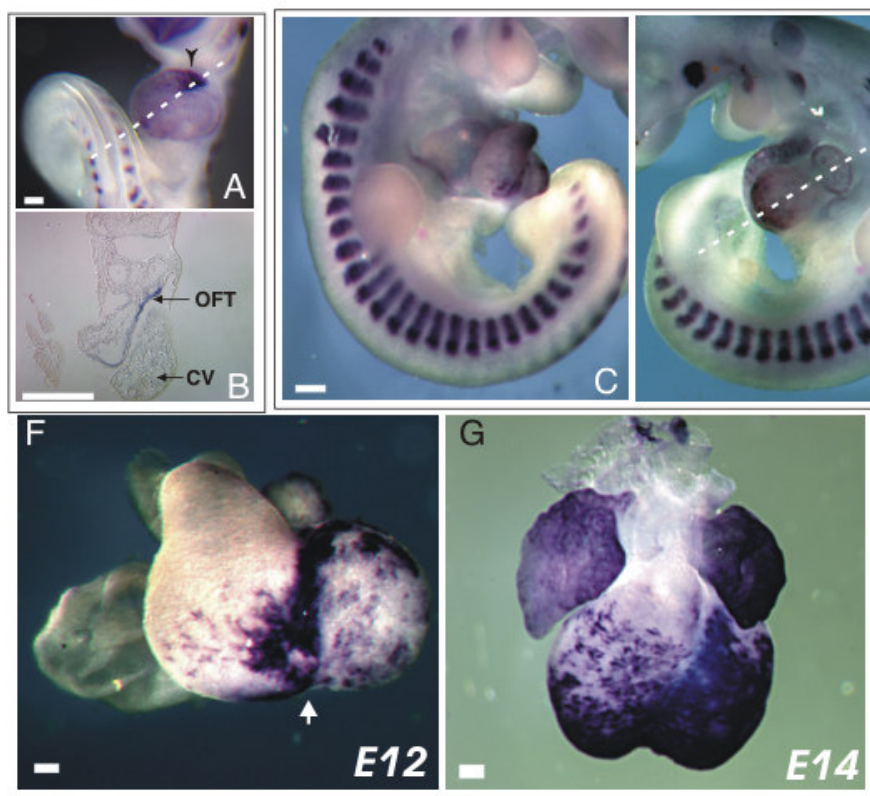

$\mathrm{D}$
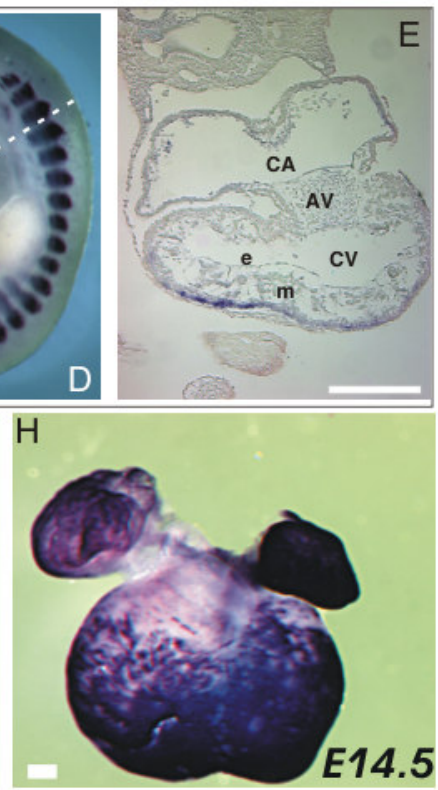

FIGURE 1. The dynamic expression of Tnnt 1 in the murine embryonic heart. WISH was performed with a Tnnt1 antisense RNA probe on E9.5-10.5 embryos (A-E) or isolated hearts E12.0-14.5 (F-H). Whole embryo samples $(A, D)$ were sectioned $(B, E)$; the white dotted line in $(A)$ and $(D)$ indicates plane of sectioning. Note the expression in the outflow track at E9.5 (A, arrowhead) and in the interventricular groove (F, arrow). Scale bar, $100 \mu \mathrm{m}$ $(\mathrm{A}, \mathrm{C}, \mathrm{F}-\mathrm{H})$ and $200 \mu \mathrm{m}(\mathrm{B}, \mathrm{E})$. AV, atrioventricular canal; CA, common atrium; CV, common ventricle; e, endocardium; m, myocardium, OFT, outflow tract.

track remain negative (Fig. $1 \mathrm{G}, \mathrm{H}$ ). The Tnnt1 atrial expression becomes prominent at E12.5, with the left atrium being more positive.

\section{Tnnt1 RNA Expression in Fog2 Null and Gata4 ${ }^{k i k i}$ Hearts}

Microarray analysis and qRT-PCR both reveal a dramatic down-regulation of Tnnt1 expression upon GATA4-FOG2 interaction loss. In accordance with microarray data, qRT-PCR demonstrated a significant down-regulation of Tnnt1 in E12.5 mutant hearts (Fig. 2A). WISH corroborated this down-regulation in GATA4-FOG2 mutants (Fig. 2B-D). The residual expression in the Fog2 null E12.5 heart (Fig. 2C) resembles the earlier ( E9.5) wild-type pattern, with positive cells persisting in the outflow track and the apical portion of the interventricular groove. No residual Tnntl expression is apparent in the atria or ventricles of the Gata4 mutant $\left(\right.$ Gata4 $\left.^{k i / k i}\right)$ at E12.5 (Fig. 2D). Importantly, the noncardiac expression of Tnnt1 (e.g., in skeletal muscle) remains intact in both Fog 2 and Gata4 mutants (data not shown).

\section{Tnnt1 Expression is Increased in $\alpha M h c-F o g 2$ Transgenic Animals}

To validate Tnnt1 as a bona fide target of the GATA4-FOG2 complex, we performed additional experiments. Fog2 expression is decreased in the developing heart shortly after E16.5[4]. Tnnt1 expression was reported to follow a similar trend[26,27]. If the GATA4-FOG2 complex is required for Tnnt1 activity, FOG2 concentration could be limiting and therefore responsible for Tnnt1 downregulation in the late gestation heart. In this case, cardiac Fog 2 overexpression should be sufficient for increasing Tnnt1 levels in the heart. To test this possibility, we took advantage of the transgenic mice that 


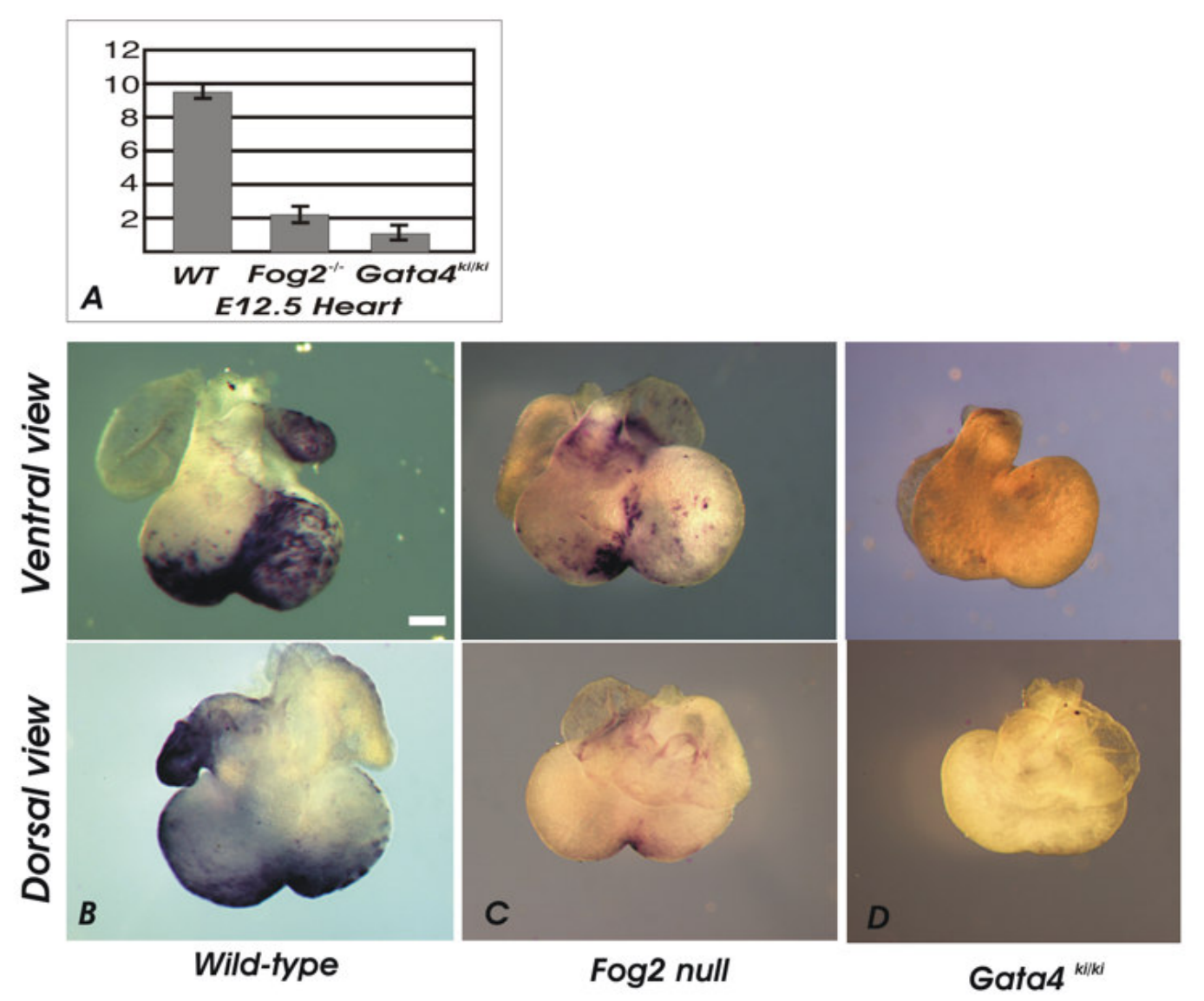

FIGURE 2. Tnnt1 expression requires GATA4-FOG2 interaction. (A) Real-time PCR analysis of the Tnnt1 gene expression in wild-type, $\mathrm{Fog}_{2}{ }^{\%}$, and $\mathrm{Gata}_{4}{ }^{k i k i} \mathrm{E} 12.5$ hearts; the y axis shows values for both genes normalized to the Gapdh RNA copy number. (B-D) WISH was performed with a Tnntl antisense RNA probe on isolated hearts from E12.5 control (B), Fog2 null (C), and Gata4 ${ }^{k i k i}$ (D) mutant embryos. Scale bar, $100 \mu \mathrm{m}$.

express Fog2 under the control of regulatory sequences from the cardiac alpha myosin heavy chain $(\alpha M h c)$ promoter[7]. The $\alpha M h c$ promoter directs expression specifically to cardiomyocytes by E10.5[28]; the $\alpha M h c-F o g 2$ transgenic animals have been previously described and were successfully used to rescue the lethality of $F o g 2^{-/}$embryos at $\sim$ E14.5 from cardiac pathology[7,19].

As was shown previously[26,27], embryonic Tnntl RNA expression is transient in the murine heart and starts to decline after E16.5. Accordingly, WISH with control E17.5 hearts showed low levels of cardiac Tnntl expression (Fig. 3A). In contrast, in the E17.5 $\alpha M h c-F o g 2$ transgenics, the Tnnt1 expression level remains high in the atria, the known preferential site of $\alpha M h c$ expression at this stage (e.g., [29]) (Fig. 3B, arrows). qRT-PCR confirms that Tnnt1 levels are elevated in the $\alpha M h c-F o g 2$ neonatal animals compared to the controls (Fig. 3C). This demonstrates that elevating Fog2 levels in cardiomyocytes is sufficient for increasing Tnntl expression.

\section{Tnnt1 Expression is Restored in Hearts with Myocardial-Restricted Fog2}

As Tnnt1 expression is increased in $\alpha M h c$-Fog 2 transgenics, we sought to determine whether myocardial FOG2 is sufficient to recapitulate (rescue) Tnnt1 cardiac expression in the otherwise Fog2-null fetuses. To test this, we crossed the $\alpha M h c-F o g 2$ animals to $F o g 2^{+/-}$mice to generate $\alpha M h c-F o g 2 ; F o g 2^{+/}$animals, 


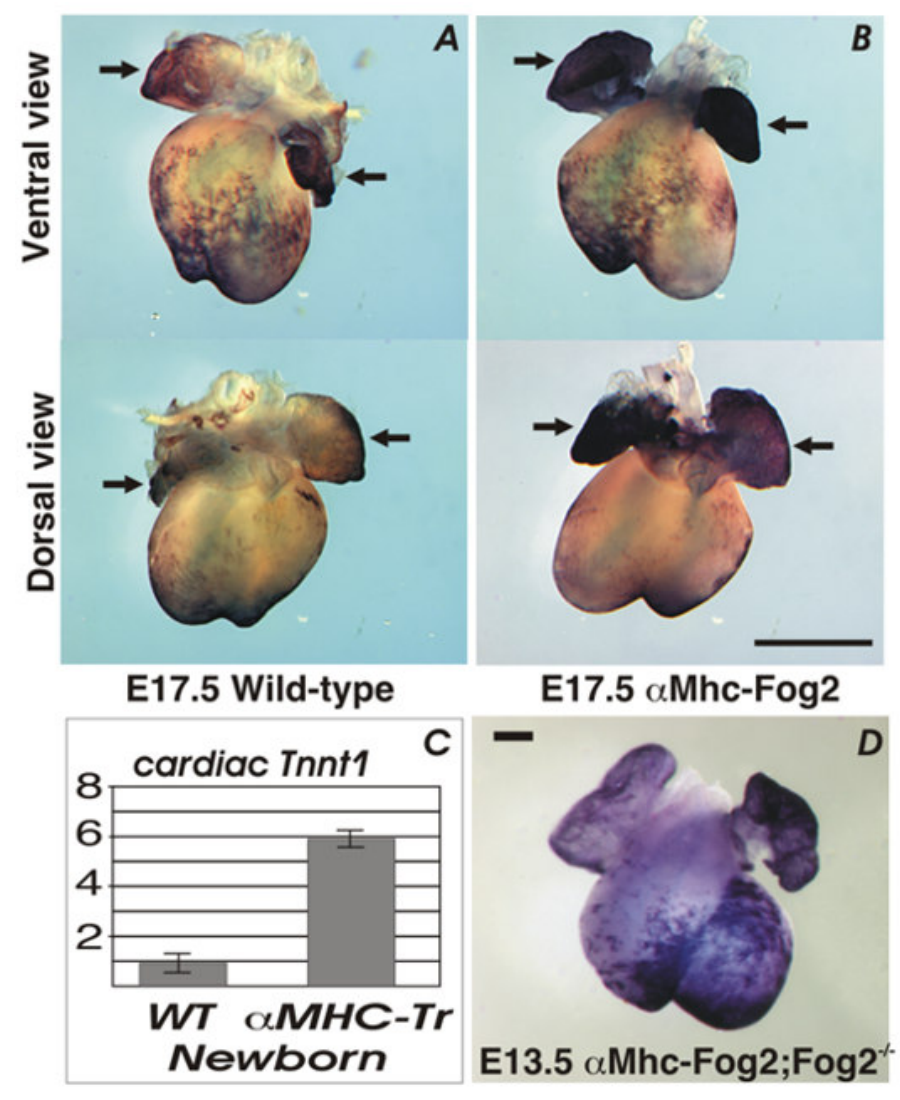

FIGURE 3. FOG2 regulates Tnnt1 expression in myocardium. (A,B) WISH was performed with Tnntl antisense RNA probe on isolated hearts from E17.5 control (A) and $\alpha M h c-F o g 2$ transgenic embryos (B). Note enhanced expression in the atria of the transgenic embryos compared to the control (arrows). (C) Real-time PCR analysis of the Tnntl gene expression in wildtype and $\alpha M h c$-Fog 2 newborn hearts; the y axis shows values for both genes normalized to the Gapdh RNA copy number. (D) WISH was performed with Tnnt1 antisense RNA probe on an isolated heart from E13.5 $\alpha M h c$ Fog $2 ; F_{o g} 2^{-}$mutant embryo; the expression pattern resembles that of the control heart (e.g., Fig. 2B) and not the Fog2 null mutant (e.g., Fig. 2C). Scale bar, $1 \mathrm{~mm}$ (B) and $100 \mu \mathrm{m}$ (D).

and backcrossed these to the $\mathrm{Fog}^{+/-}$animals to obtain and examine the $\alpha M h c-F o g 2 ; F o g 2^{-/}$fetuses. During mid-gestation, $\alpha M h c$ promoter directs expression to the ventricular myocardium[28]; hence, the $\alpha M h c-F o g 2 / F o g 2^{--}$fetuses express Fog 2 cDNA driven by the $\alpha M h c$ promoter exclusively in the myocardium and are otherwise Fog2 null. In the E13.5 hearts from these "rescued" embryos, the Tnnt1 expression pattern is now restored (Fig. 3D) and appears indistinguishable from that in the contemporaneous wild-type hearts (compare Fig. 3D to Fig. 2B). We conclude that, although Fog2 is expressed in all three cardiac layers, restoring FOG2 function specifically in the myocardium is sufficient to "rescue" Tnntl expression.

\section{Proximal DNA Elements are Sufficient to Direct both Skeletal and Cardiac Tnnt1 Expression}

The cis-elements that are required to drive Tnnt1 expression in skeletal muscle have not been defined[30]; even less is known about the transcriptional regulation of this gene in the heart. The genomic organization 
of the human and mouse Tnntl gene has been reviewed[30]. The interesting feature of the Tnntl gene is its location in very close proximity to cardiac-restricted troponin I (Tnni3); the distance between the Tnnt1 and Tnni3 is only $2.4 \mathrm{~kb}$ in the mouse (2.6 kb in the human)[30]. Intriguingly, the gene downstream of Tnnt1 (14.7 kb; Ppp1r12c, Mbs85) is also highly expressed in the hearts of mice[31] and men[32]. However, both of these genetic neighbors are expressed normally in the Gata4-Fog2 mutants, excluding the possibility of coregulation (not shown).

Inspection of the Tnnt1 genomic locus using an ECR (evolutionary conserved region) browser[33] confirmed previously reported genomic organization of the locus[30]; however, outside of the TNNT1 coding sequence, we detected little conservation even between mammals, with the exception of the phylogenetically conserved fifth intron (Supplemental Fig. 1). This was unexpected, as a similar pattern and timing of cardiac expression was reported for a human and rat gene[34] and, hence, a better conservation of the regulatory sequences could have been expected. In order to identify the cis-regulatory elements that are responsible for cardiac-specific regulation of the Tnnt1 gene by the GATA4-FOG2 complex, we generated a $\mathrm{LacZ}$ (bacterial $\beta$-galactosidase) fusion transgenic construct (Fig. 4A). The construct contained the 2.4-kb region upstream of the Tnnt1 transcription start site with the 5' boundary delimited by the Tnni3 ORF; we also inserted the phylogenetically conserved fifth intron 3' to the LacZ-SV40polyA cassette to generate 2.4Tnnt1-LacZpa-I5 (Fig. 4A). Transgenic construct was injected into fertilized eggs, and $\mathrm{F}_{0}$ transgenic embryos were collected and analyzed at E12.5. The 2.4-Tnnt1-LacZpa-I5 sequences were fully sufficient to drive the expression of the LacZ reporter in the somites and developing skeletal muscle in all transgenic embryos (Fig. 4B). In addition to skeletal expression, we also observed cardiac-specific expression of the lacZ reporter in some (but not all) of these $\mathrm{F}_{0}$ E12.5 embryos (Fig. 4C).
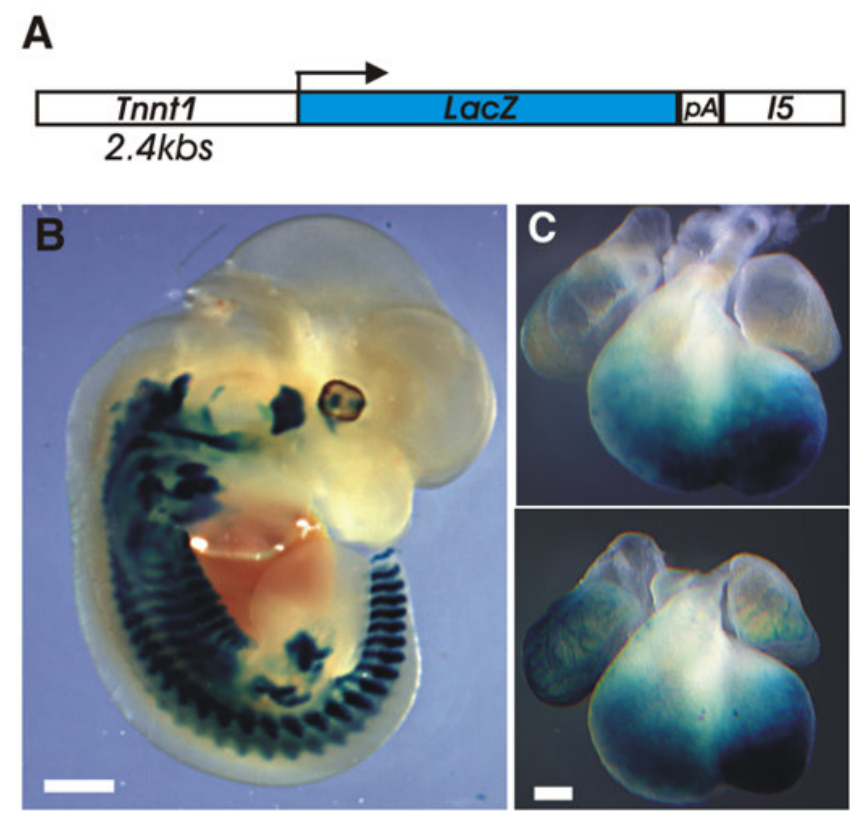

FIGURE 4. The Tnnt1 regulatory elements direct reporter expression to skeletal and cardiac tissue. (A) A diagram of the Tnnt1-LacZ transgenic construct. (B,C) Whole-mount X-gal staining of the E12.5 transgenic embryo (B) and X-gal stained hearts from two different transgenic embryos (C). Compare the staining in (C) to the Tnntl WISH-stained heart in Fig. 1. Scale bar, $200 \mu \mathrm{m}$ (B) and $100 \mu \mathrm{m}(\mathrm{C})$

While it is possible that other transcription factors are responsible for Tnnt1 regulation, we have not identified any myocardially expressed transcription factors in our microarray experiments. Hence, we 
reasoned that the GATA4-FOG2 complex is regulating Tnnt1 expression directly through one of the GATA/TATC sequences. Despite numerous attempts, a chromatin immunoprecipitation (ChIP) assay from the E12.5 embryonic hearts did not pull down DNA containing GATA sites within Tnnt1 DNA (not shown). Both antibodies used in this experiment ( $\alpha$ GATA4 or $\alpha$ FOG2) were previously used successfully to isolate GATA-containing elements in the $L h x 9$ regulatory region[25]. Experiments are currently in progress to address the mechanism of the GATA4-FOG2-dependent regulation of the Tnnt1 by using the ES cell in vitro differentiation system.

\section{DISCUSSION}

Interaction between GATA4 and FOG2 is required in normal cardiac development; however, the genetic mechanism of GATA4 and/or FOG2 action in the heart, and specifically in the myocardium, is not well understood. It has even been proposed that the myocardial defects in Gata4 null hearts may be secondary to GATA4 loss in the proepicardium[15].

We now identify the Tnnt1 gene as a target of the GATA4-FOG2 complex in the myocardium. The cis-acting elements that are required to drive this gene's expression in skeletal muscle have not been defined[30]; we now show that the $2.4 \mathrm{~kb}$ and sequences from intron 5 are sufficient to direct musclespecific expression during mouse embryonic development. While all transgenic embryos express the transgene in the skeletal muscle, cardiac expression was observed in two out of five embryos, suggesting that while additional elements are required for consistent expression in the heart, sequences necessary for Tnnt1 cardiac expression are present within the transgene's regulatory elements. Despite our repeated attempts, the cardiac ChIP assay could not detect a GATA4-FOG2 complex bound to GATA/TATC elements within these regulatory sequences. In light of these negative results, we conclude that Tnnt 1 is unlikely to be directly regulated by the GATA4-FOG2 complex in the heart.

A 2.4Tnnt1-LacZpA-I5 reporter we have generated is robustly expressed in embryonic skeletal muscle, thus indicating that the 2.4-kb fragment and intron 5 contain all the elements necessary for skeletal muscle expression. As skeletal muscle does not express FOG molecules, Tnnt1 expression in this tissue has to be independent of GATA-FOG interaction; correspondingly, Tnntl is expressed normally in skeletal muscle of the Fog 2 null and Gata4 $4^{k i k i}$ mutants. Outside of skeletal muscle, Tnnt1 RNA has been detected in several other tissues; the significance of this extraskeletal Tnntl gene expression and its transcriptional regulation are not understood. In the murine and human heart, Tnntl RNA expression was previously described; this expression is transient during embryogenesis and starts to decline in mice after E16.5[26,27]. Expression of TNNT1 was also reported in several examined human embryonic stem cell lines (where it is lost upon differentiation[35]) and in aging hearts[36,37]. Furthermore, Tnnt1 expression is dramatically induced in brains (neurons) of mice treated with ketamine[38]. Expression of Tnnt1 outside of muscle tissue hints at alternative function other than its conventional structural role in the sarcomere.

In humans, intact TNNT1 in skeletal musculature is required to support life: a nonsense mutation in TNNT1 causes an autosomal-recessive Amish Nemaline Myopathy (ANM). The children affected by ANM die of respiratory insufficiency, usually in their second year of life. The mutation (a stop codon) in exon 11 results in a deletion of the last 83 amino acids of the protein, removing the protein-binding modules that are necessary for TNNT1's structural function[39]. Although congestive heart failure has been commonly observed for the ANM patients, no evidence of primary cardiac involvement was reported[39].

While elucidating the function of Tnnt1 (sTnT) in mammalian development and cardiogenesis will have to await this gene's deletion in mice, targeted disruption of its cardiac homologue Tnnt2 (cTnT) is embryonic lethal at around E10[40,41]. It has been reported that, while nearly lacking a heartbeat, a minor twitching consisting of a few cardiomyocytes was observed in all of E10 and about half of E9 $c T n T^{/-}$ $\left(\right.$ Tnnt $\left.2^{-1}\right)$ embryos, suggesting that some developmentally regulated mechanism compensated partially for the lack of TNNT2[41]. Interestingly, the beating cells were observed in the outflow tract in these embryos, which is the zone of Tnntl expression (Fig. 2A), in line with the author's hypothesis that Tnnt1 is able to compensate for Tnnt2 at this stage. 


\section{SUPPLEMENTARY MATERIAL}

SUPPLEMENTAL TABLE 1

Differentially Expressed Genes in E12.5 Control vs. Gata4 ${ }^{k i k i}$ Hearts

\begin{tabular}{|c|c|c|c|c|c|}
\hline $\begin{array}{l}\text { Gene } \\
\text { Order }\end{array}$ & $\begin{array}{l}\text { Affymetrix } \\
\text { Probe }\end{array}$ & $\begin{array}{l}\text { Fold } \\
\text { Change }\end{array}$ & $\begin{array}{l}\text { Sequence } \\
\text { Accession } \\
\text { Number }\end{array}$ & $\begin{array}{l}\text { Gene } \\
\text { Symbol }\end{array}$ & Gene Name \\
\hline \multicolumn{6}{|c|}{ Downregulated genes } \\
\hline 1. & 1426561_a_at & -5.81 & NM 001029836.1 & Npnt & Nephronectin \\
\hline 2. & 1419606_a_at & -5.77 & NM 011618.1 & Tnnt1 & Troponin T1 \\
\hline 3. & 1428549_at & -5.48 & NM 028804.1 & Ccdc3 & Coiled-coil domain containing 3 \\
\hline 4. & 1417023_a_at & -5.3 & NM 024406.2 & Fabp4 & Fatty acid binding protein 4 , adipocyte \\
\hline 5. & 1421144_at & -5.05 & NM 023879.2 & Rpgrip1 & $\begin{array}{l}\text { Retinitis pigmentosa GTPase regulator } \\
\text { interacting protein } 1\end{array}$ \\
\hline 6. & 1452088_at & -4.15 & NM 028106.2 & Zbed3 & Zinc finger, BED domain containing 3 \\
\hline 7. & 1427457_a_at & -4.13 & NM 009755.2 & $B m p 1$ & Bone morphogenetic protein 1 \\
\hline 8. & 1423062_at & -4.08 & NM 008343.2 & $\operatorname{lgfbp3}$ & $\begin{array}{l}\text { Insulin-like growth factor binding protein } \\
3\end{array}$ \\
\hline 9. & 1416515_at & -3.97 & NM 007984.2 & Fscn1 & $\begin{array}{l}\text { Fascin homolog 1, actin bundling } \\
\text { protein (Strongylocentrotus } \\
\text { purpuratus) }\end{array}$ \\
\hline 10. & 1427476_a_at & -3.96 & $\underline{\mathrm{NM}} 053084.1$ & Trim32 & Tripartite motif protein 32 \\
\hline 11. & 1417860_a_at & -3.95 & NM 133903.2 & Spon2 & Spondin 2, extracellular matrix protein \\
\hline 12. & 1460232_s_at & -3.9 & NM 013821.3 & $\begin{array}{l}H s d 3 b 2 / / I \\
H s d 3 b 6\end{array}$ & $\begin{array}{l}\text { Hydroxysteroid dehydrogenase-2, } \\
\text { delta }<5>-3 \text {-beta /hydroxysteroid } \\
\text { dehydrogenase-6, delta }<5>-3 \text {-beta } 1\end{array}$ \\
\hline 13. & 1436399_s_at & -3.89 & NM 013724.2 & Nrk & Nik related kinase \\
\hline 14. & 1417888_at & -3.81 & NM 023233.2 & Trim13 & Tripartite motif protein 13 \\
\hline 15. & 1427385_s_at & -3.78 & NM 011501.1 & Strm & Striamin \\
\hline 16. & 1416033_at & -3.67 & NM 134142.1 & Tmem109 & Transmembrane protein 109 \\
\hline 17. & 1449319_at & -3.66 & NM 138683.2 & Rspondin & $\begin{array}{l}\text { Thrombospondin type } 1 \text { domain } \\
\text { containing gene }\end{array}$ \\
\hline 18. & 1448147_at & -3.63 & NM 013869.4 & Tnfrsf19 & $\begin{array}{l}\text { Tumor necrosis factor receptor } \\
\text { superfamily, member } 19\end{array}$ \\
\hline 19. & 1418908_at & -3.62 & NM 013626.3 & Pam & $\begin{array}{l}\text { Peptidylglycine alpha-amidating } \\
\text { monooxygenase }\end{array}$ \\
\hline 20. & 1451331_at & -3.46 & NM 144828.1 & Ppp1r1b & $\begin{array}{l}\text { Protein phosphatase 1, regulatory } \\
\text { (inhibitor) subunit 1B }\end{array}$ \\
\hline 21. & 1418723_at & -3.35 & NM 022983.3 & Edg7 & $\begin{array}{l}\text { Endothelial differentiation, } \\
\text { lysophosphatidic acid G protein- } \\
\text { coupled receptor } 7\end{array}$ \\
\hline 22. & 1438936_s_at & -3.35 & $\underline{\mathrm{NM}} 009640.3$ & Ang1 & Angiopoietin 1 \\
\hline 23. & 1418535_at & -3.32 & NM 016846.3 & $R g / 1$ & $\begin{array}{l}\text { Ral guanine nucleotide dissociation } \\
\text { stimulator,-like } 1\end{array}$ \\
\hline 24. & 1451203_at & -3.28 & NM 013593.2 & $M b$ & Myoglobin \\
\hline 25. & 1437733_at & -3.25 & NM 010124.2 & Eif4ebp2 & $\begin{array}{l}\text { Eukaryotic translation initiation factor } 4 \mathrm{E} \\
\text { binding protein } 2\end{array}$ \\
\hline
\end{tabular}




\section{SUPPLEMENTAL TABLE 1 (continued)}

\begin{tabular}{|c|c|c|c|c|c|}
\hline $\begin{array}{l}\text { Gene } \\
\text { Order }\end{array}$ & $\begin{array}{l}\text { Affymetrix } \\
\text { Probe }\end{array}$ & $\begin{array}{l}\text { Fold } \\
\text { Change }\end{array}$ & $\begin{array}{l}\text { Sequence } \\
\text { Accession } \\
\text { Number }\end{array}$ & $\begin{array}{l}\text { Gene } \\
\text { Symbol }\end{array}$ & Gene Name \\
\hline \multicolumn{6}{|c|}{ Upregulated genes } \\
\hline 1. & 1454866_s_at & 59.81 & NM 172469.3 & Clic6 & Chloride intracellular channel 6 \\
\hline 2. & 1433930_at & 43.92 & NM 152803.4 & Hpse & Heparanase \\
\hline 3. & 1416645_a_at & 39.08 & NM 007423.4 & Afp & Alpha fetoprotein \\
\hline 4. & 1434165_at & 22.67 & NM 172469.3 & Clic6 & Chloride intracellular channel 6 \\
\hline 5. & 1416646_at & 13.07 & NM 007423.4 & Afp & Alpha fetoprotein \\
\hline 6. & 1427119_at & 11.21 & $\overline{N M} 011463.2$ & Spink4 & Serine protease inhibitor, Kazal type 4 \\
\hline 7. & 1416468_at & 8.5 & NM 013467.3 & Aldh1a1 & $\begin{array}{l}\text { Aldehyde dehydrogenase family } 1 \text {, } \\
\text { subfamily A } 1\end{array}$ \\
\hline 8. & 1418199_at & 6.92 & NM 053149.2 & Hemgn & Hemogen \\
\hline 9. & 1460214_at & 5.9 & NM 008791.2 & Pcp4 & Purkinje cell protein 4 \\
\hline 10. & 1420664_s_at & 5.47 & NM 011171.1 & Procr & Protein C receptor, endothelial \\
\hline 11. & 1422836_at & 5.36 & NM 134163.4 & Mbn/3 & Muscleblind-like 3 (Drosophila) \\
\hline 12. & 1423691_x_at & 5.32 & NM 031170.2 & Krt2-8 & Keratin complex 2 , basic, gene 8 \\
\hline 13. & 1420647_a_at & 5.19 & NM 031170.2 & Krt2-8 & Keratin complex 2 , basic, gene 8 \\
\hline 14. & 1429159_at & 4.93 & $\underline{\text { AK014514.1 }}$ & $\begin{array}{l}46314080 \\
11 R i k\end{array}$ & RIKEN cDNA 4631408011 gene \\
\hline 15. & 1449169_at & 4.84 & NM 008216.3 & Has2 & Hyaluronan synthase 2 \\
\hline 16. & 1427428_at & 4.08 & NM 029465.3 & Clec4g & $\begin{array}{l}\text { C-type lectin domain family } 4 \text {, member } \\
\mathrm{g}\end{array}$ \\
\hline 17. & 1435989_x_at & 4.02 & NM 031170.2 & Krt2-8 & Keratin complex 2 , basic, gene 8 \\
\hline 18. & 1428942_at & 3.83 & NM 008630.2 & Mt2 & Metallothionein 2 \\
\hline 19. & 1418678_at & 3.69 & $\overline{N M ~ 008216.3}$ & Has2 & Hyaluronan synthase 2 \\
\hline 20. & 1456014_s_at & 3.69 & NM 153795.1 & Fermt3 & Fermitin family homolog 3 (Drosophila) \\
\hline 21. & 1423429_at & 3.59 & NM 008818.2 & Rhox5 & Reproductive homeobox 5 \\
\hline 22. & 1429146_at & 3.54 & NM 001160345.1 & Svip & Small VCP/p97-interacting protein \\
\hline 23. & 1455599_at & 3.5 & NM 001033399.4 & Gfod1 & $\begin{array}{l}\text { Glucose-fructose oxidoreductase } \\
\text { domain containing } 1\end{array}$ \\
\hline 24. & 1449425_at & 3.47 & NM 023653.4 & Wnt2 & $\begin{array}{l}\text { Wingless-related MMTV integration site } \\
2\end{array}$ \\
\hline 25. & 1427183_at & 3.45 & NM 146015.2 & Efemp1 & $\begin{array}{l}\text { Epidermal growth factor-containing } \\
\text { fibulin-like extracellular matrix protein } \\
1\end{array}$ \\
\hline
\end{tabular}

Calculated by the Gene traffic program.

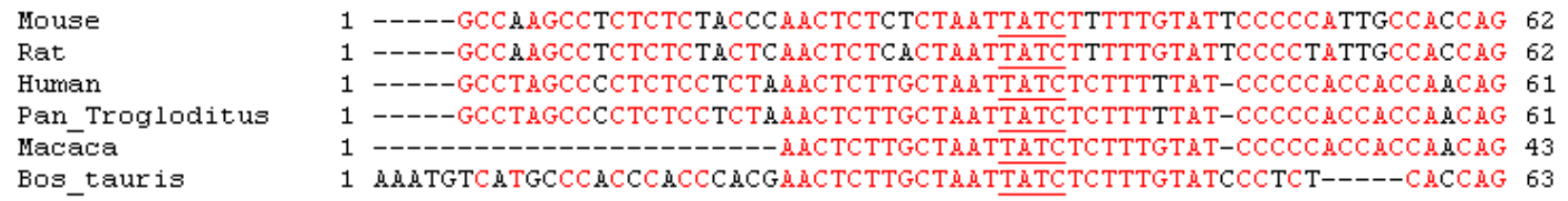

SUPPLEMENTAL FIGURE 1. The alignment of the phylogenetically conserved Tnnt1 intron5 sequences from six mammalian species; the position of the conserved TATC/GATA element is underlined. 


\section{ACKNOWLEDGMENTS}

The authors would like to thank the Dartmouth Transgenic and Genetic Construct Shared Resource Center for their help in generating the transient transgenic animals; we also want to thank Ms. Shawna Fox for proofreading. This work was supported in part by the NIH (R01HD42751, NICHD) award to SGT.

\section{REFERENCES}

1. Cantor, A.B. and Orkin, S.H. (2005) Coregulation of GATA factors by the Friend of GATA (FOG) family of multitype zinc finger proteins. Semin. Cell Dev. Biol. 16, 117-128.

2. Sorrentino, R.P., Gajewski, K.M., and Schulz, R.A. (2005) GATA factors in Drosophila heart and blood cell development. Semin. Cell Dev. Biol. 16, 107-116.

3. Lu, J.R., McKinsey, T.A., Xu, H., Wang, D.Z., Richardson, J.A., and Olson, E.N. (1999) FOG-2, a heart- and brainenriched cofactor for GATA transcription factors. Mol. Cell Biol. 19, 4495-4502.

4. Svensson, E.C., Tufts, R.L., Polk, C.E., and Leiden, J.M. (1999) Molecular cloning of FOG-2: a modulator of transcription factor GATA-4 in cardiomyocytes. Proc. Natl. Acad. Sci. U. S. A. 96, 956-961.

5. Tevosian, S.G., Deconinck, A.E., Cantor, A.B., Rieff, H.I., Fujiwara, Y., Corfas, G., and Orkin, S.H. (1999) FOG-2: a novel GATA-family cofactor related to multitype zinc-finger proteins Friend of GATA-1 and U-shaped. Proc. Natl. Acad. Sci. U. S. A. 96, 950-955.

6. Svensson, E.C., Huggins, G.S., Lin, H., Clendenin, C., Jiang, F., Tufts, R., Dardik, F.B., and Leiden, J.M. (2000) A syndrome of tricuspid atresia in mice with a targeted mutation of the gene encoding Fog-2. Nat. Genet. 25, 353-356.

7. Tevosian, S.G., Deconinck, A.E., Tanaka, M., Schinke, M., Litovsky, S.H., Izumo, S., Fujiwara, Y., and Orkin, S.H. (2000) FOG-2, a cofactor for GATA transcription factors, is essential for heart morphogenesis and development of coronary vessels from epicardium. Cell 101, 729-739.

8. $\quad$ Novak, K. (2000) Lost in the FOG. Nat. Med. 6, 864.

9. Shalaby, F., Rossant, J., Yamaguchi, T.P., Breitman, M.L., and Schuh, A.C. (1995) Failure of blood island formation and vasculogenesis in flk-1 deficient mice. Nature 376, 62-66.

10. Bautch, V.L. and Ambler, C.A. (2004) Assembly and patterning of vertebrate blood vessels. Trends Cardiovasc. Med. 14, 138-143.

11. Kuo, C.T., Morrisey, E.E., Anandappa, R., Sigrist, K., Lu, M.M., Parmacek, M.S., Soudais, C., and Leiden, J.M. (1997) GATA4 transcription factor is required for ventral morphogenesis and heart tube formation. Genes Dev. 11, 1048-1060.

12. Molkentin, J.D., Lin, Q., Duncan, S.A., and Olson, E.N. (1997) Requirement of the transcription factor GATA4 for heart tube formation and ventral morphogenesis. Genes Dev. 11, 1061-1072.

13. Narita, N., Bielinska, M., and Wilson, D.B. (1997) Cardiomyocyte differentiation by GATA-4-deficient embryonic stem cells. Development 124, 3755-3764.

14. Crispino, J.D., Lodish, M.B., Thurberg, B.L., Litovsky, S.H., Collins, T., Molkentin, J.D., and Orkin, S.H. (2001) Proper coronary vascular development and heart morphogenesis depend on interaction of GATA-4 with FOG cofactors. Genes Dev. 15, 839-844.

15. Watt, A.J., Battle, M.A., Li, J., and Duncan, S.A. (2004) GATA4 is essential for formation of the proepicardium and regulates cardiogenesis. Proc. Natl. Acad. Sci. U. S. A. 101, 12573-12578.

16. Oka, T., Maillet, M., Watt, A.J., Schwartz, R.J., Aronow, B.J., Duncan, S.A., and Molkentin, J.D. (2006) Cardiacspecific deletion of Gata4 reveals its requirement for hypertrophy, compensation, and myocyte viability. Circ. Res. 98, 837-845.

17. Zeisberg, E.M., Ma, Q., Juraszek, A.L., Moses, K., Schwartz, R.J., Izumo, S., and Pu, W.T. (2005) Morphogenesis of the right ventricle requires myocardial expression of Gata4. J. Clin. Invest. 115, 1522-1531.

18. Garg, V., Kathiriya, I.S., Barnes, R., Schluterman, M.K., King, I.N., Butler, C.A., Rothrock, C.R., Eapen, R.S., Hirayama-Yamada, K., Joo, K., Matsuoka, R., Cohen, J.C., and Srivastava, D. (2003) GATA4 mutations cause human congenital heart defects and reveal an interaction with TBX5. Nature 6, 6.

19. Tevosian, S.G., Albrecht, K.H., Crispino, J.D., Fujiwara, Y., Eicher, E.M., and Orkin, S.H. (2002) Gonadal differentiation, sex determination and normal Sry expression in mice require direct interaction between transcription partners GATA4 and FOG2. Development 129, 4627-4634.

20. Chee, M., Yang, R., Hubbell, E., Berno, A., Huang, X.C., Stern, D., Winkler, J., Lockhart, D.J., Morris, M.S., and Fodor, S.P. (1996) Accessing genetic information with high-density DNA arrays. Science 274, 610-614.

21. Lipshutz, R.J., Fodor, S.P., Gingeras, T.R., and Lockhart, D.J. (1999) High density synthetic oligonucleotide arrays. Nat. Genet. 21, 20-24.

22. Adameyko, I.I., Mudry, R.E., Houston-Cummings, N.R., Veselov, A.P., Gregorio, C.C., and Tevosian, S.G. (2005) Expression and regulation of mouse SERDIN1, a highly conserved cardiac-specific leucine-rich repeat protein. Dev. 
Dyn. 233, 540-552.

23. Wilkinson, D.G. (1992) In Situ Hybridization. IRL Press, Oxford.

24. Manuylov, N.L., Manuylova, E., Avdoshina, V., and Tevosian, S. (2008) Serdin1/Lrrc10 is dispensable for mouse development. Genesis 46, 441-446.

25. Smagulova, F.O., Manuylov, N.L., Leach, L.L., and Tevosian, S.G. (2008) GATA4/FOG2 transcriptional complex regulates Lhx9 gene expression in murine heart development. BMC Dev. Biol. 8, 67.

26. Krishan, K., Morgan, M.J., Zhao, W., and Dhoot, G.K. (2000) Slow troponin T mRNA in striated muscles is expressed in both cell type and developmental stage specific manner. J. Muscle Res. Cell Motil. 21, 527-536.

27. Wang, Q., Reiter, R.S., Huang, Q.Q., Jin, J.P., and Lin, J.J. (2001) Comparative studies on the expression patterns of three troponin $\mathrm{T}$ genes during mouse development. Anat. Rec. 263, 72-84.

28. Subbarayan, V., Mark, M., Messadeq, N., Rustin, P., Chambon, P., and Kastner, P. (2000) RXRalpha overexpression in cardiomyocytes causes dilated cardiomyopathy but fails to rescue myocardial hypoplasia in RXRalpha-null fetuses. J. Clin. Invest. 105, 387-394.

29. Palermo, J., Gulick, J., Colbert, M., Fewell, J., and Robbins, J. (1996) Transgenic remodeling of the contractile apparatus in the mammalian heart. Circ. Res. 78, 504-509.

30. Cullen, M.E., Dellow, K.A., and Barton, P.J. (2004) Structure and regulation of human troponin genes. Mol. Cell. Biochem. 263, 81-90.

31. Dutheil, N., Yoon-Robarts, M., Ward, P., Henckaerts, E., Skrabanek, L., Berns, K.I., Campagne, F., and Linden, R.M. (2004) Characterization of the mouse adeno-associated virus AAVS1 ortholog. J. Virol. 78, 8917-8921.

32. Tan, I., Ng, C.H., Lim, L., and Leung, T. (2001) Phosphorylation of a novel myosin binding subunit of protein phosphatase 1 reveals a conserved mechanism in the regulation of actin cytoskeleton. J. Biol. Chem. 276, 2120921216 .

33. Ovcharenko, I., Nobrega, M.A., Loots, G.G., and Stubbs, L. (2004) ECR Browser: a tool for visualizing and accessing data from comparisons of multiple vertebrate genomes. Nucleic Acids Res. 32, W280-286.

34. Barton, P.J., Felkin, L.E., Koban, M.U., Cullen, M.E., Brand, N.J., and Dhoot, G.K. (2004) The slow skeletal muscle troponin T gene is expressed in developing and diseased human heart. Mol. Cell. Biochem. 263, 91-97.

35. Bhattacharya, B., Miura, T., Brandenberger, R., Mejido, J., Luo, Y., Yang, A.X., Joshi, B.H., Ginis, I., Thies, R.S., Amit, M., Lyons, I., Condie, B.G., Itskovitz-Eldor, J., Rao, M.S., and Puri, R.K. (2004) Gene expression in human embryonic stem cell lines: unique molecular signature. Blood 103, 2956-2964.

36. Butte, A.J. and Kohane, I.S. (2006) Creation and implications of a phenome-genome network. Nat. Biotechnol. 24, $55-62$.

37. Lee, C.K., Allison, D.B., Brand, J., Weindruch, R., and Prolla, T.A. (2002) Transcriptional profiles associated with aging and middle age-onset caloric restriction in mouse hearts. Proc. Natl. Acad. Sci. U. S. A. 99, 14988-14993.

38. Lowe, X.R., Lu, X., Marchetti, F., and Wyrobek, A.J. (2007) The expression of Troponin T1 gene is induced by ketamine in adult mouse brain. Brain Res. 1174, 7-17.

39. Johnston, J.J., Kelley, R.I., Crawford, T.O., Morton, D.H., Agarwala, R., Koch, T., Schaffer, A.A., Francomano, C.A., and Biesecker, L.G. (2000) A novel nemaline myopathy in the Amish caused by a mutation in troponin T1. Am. J. Hum. Genet. 67, 814-821.

40. Ahmad, F., Banerjee, S.K., Lage, M.L., Huang, X.N., Smith, S.H., Saba, S., Rager, J., Conner, D.A., Janczewski, A.M., Tobita, K., Tinney, J.P., Moskowitz, I.P., Perez-Atayde, A.R., Keller, B.B., Mathier, M.A., Shroff, S.G., Seidman, C.E., and Seidman, J.G. (2008) The role of cardiac troponin T quantity and function in cardiac development and dilated cardiomyopathy. PLOS ONE 3, e2642.

41. Nishii, K., Morimoto, S., Minakami, R., Miyano, Y., Hashizume, K., Ohta, M., Zhan, D.Y., Lu, Q.W., and Shibata, Y. (2008) Targeted disruption of the cardiac troponin T gene causes sarcomere disassembly and defects in heartbeat within the early mouse embryo. Dev. Biol. 322, 65-73.

\section{This article should be cited as follows:}

Manuylov, N.L. and Tevosian, S.G. (2009) Cardiac expression of Tnnt1 requires the GATA4-FOG2 transcription complex. TheScientificWorldJOURNAL: TSW Development \& Embryology. 9, 575-587. DOI 10.1100/tsw.2009.75. 


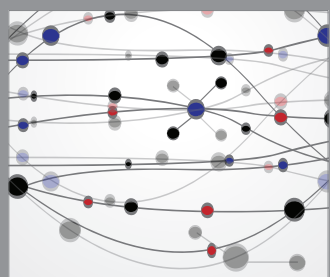

The Scientific World Journal
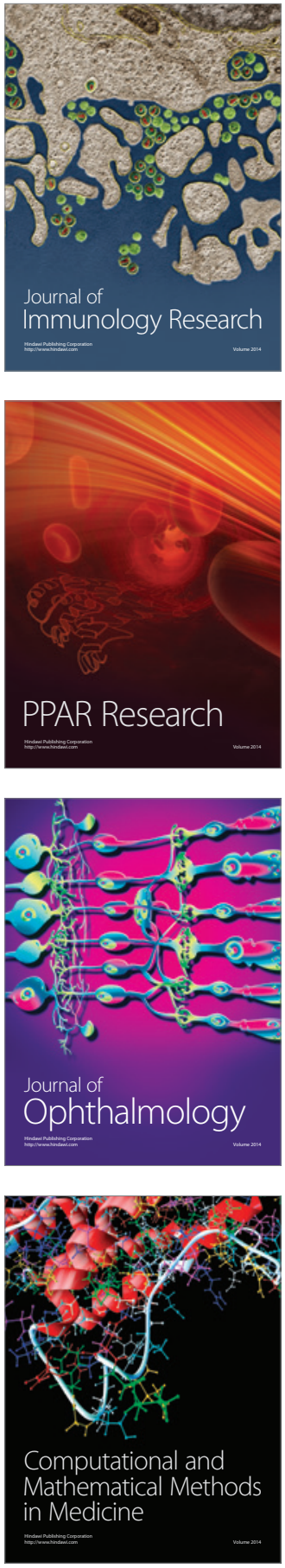

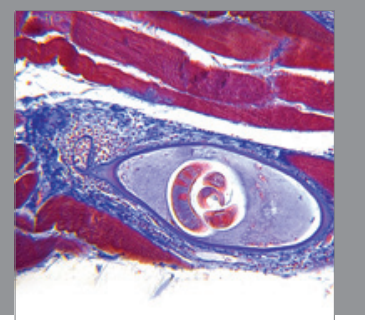

Gastroenterology

Research and Practice
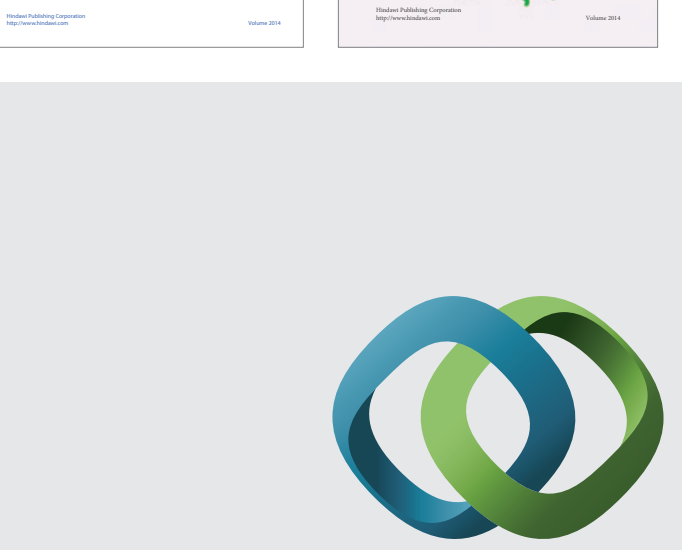

\section{Hindawi}

Submit your manuscripts at

http://www.hindawi.com
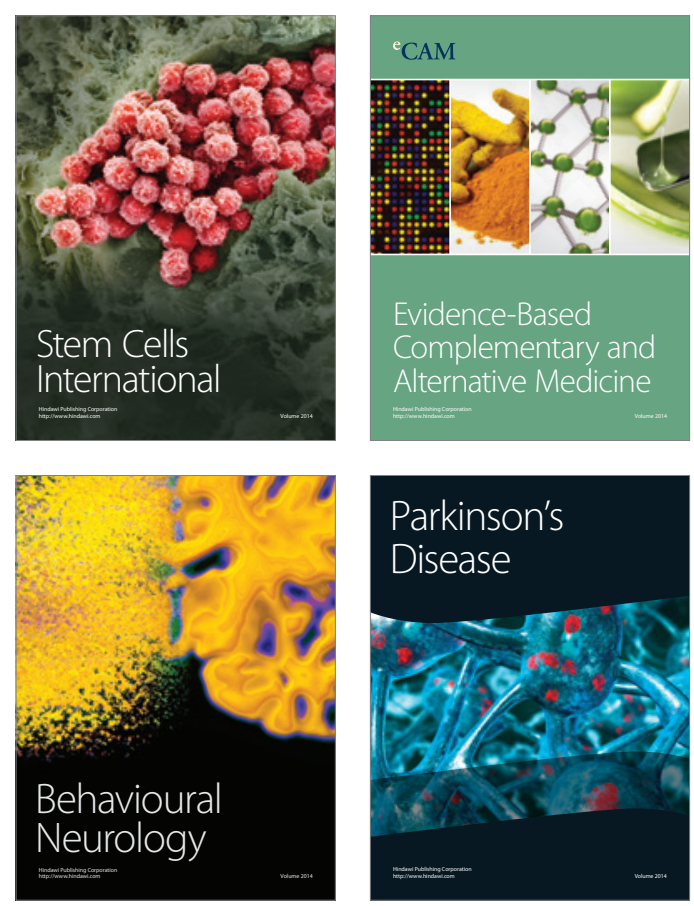

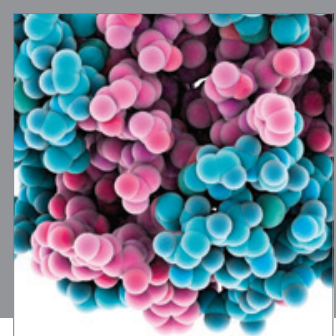

Journal of
Diabetes Research

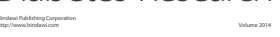

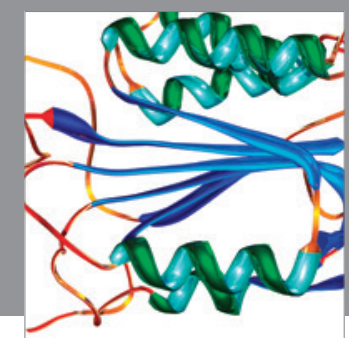

Disease Markers
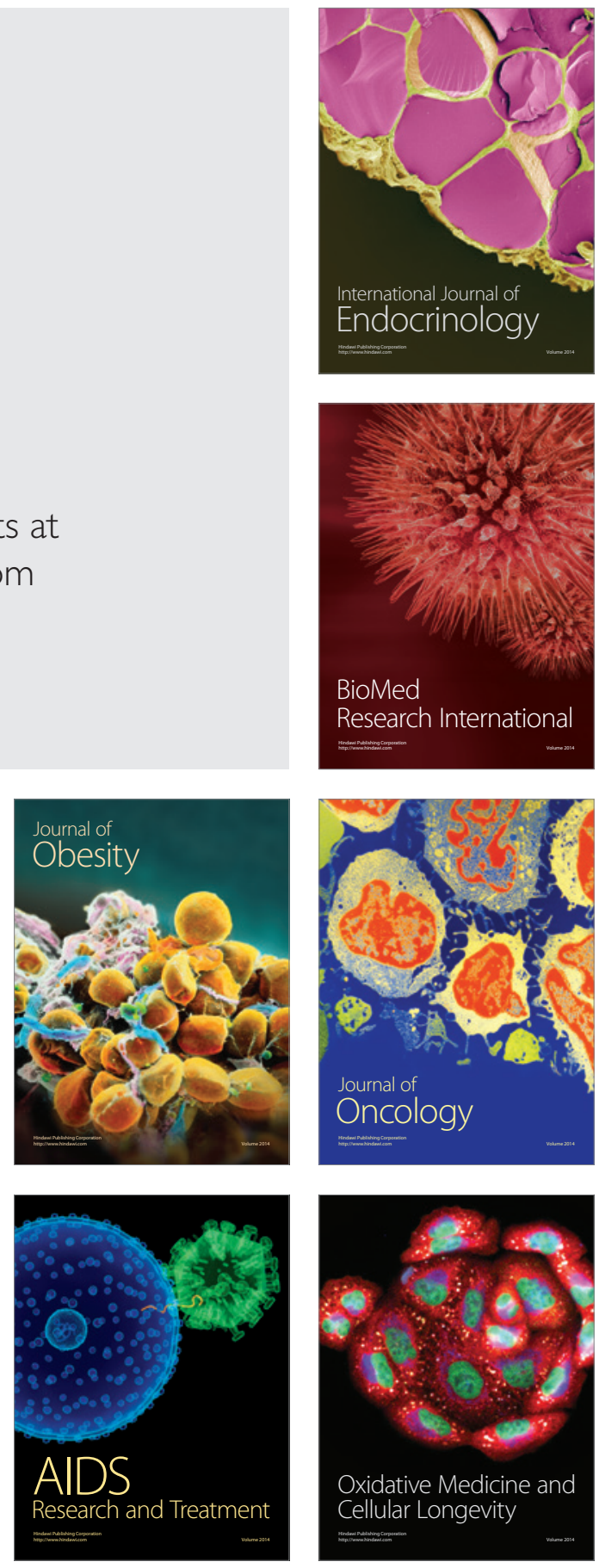